\section{- Controversy over agricultural biotechnology continues}

In the United States, some 70 million acres of transgenic crops were grown in 1999 (out of 98.6 million transgenic acres globally), and an estimated two-thirds of the U.S. food supply now contains genetically modified ingredients. While grower adoption of herbicide- and insectresistant crops has been rapid and widespread since their introduction in 1995, controversies have arisen at home and abroad concerning how transgenic crops and foods will be regulated, traded and labeled in the 21st century.

For example, Japan, South Korea, Australia and the European Union are working on laws requiring mandatory labeling of food products containing transgenic ingredients. Meanwhile, European demand for U.S. soybeans and corn has declined, resulting in a significant decline in exports. Bills requiring labeling of genetically modified organisms have been introduced in the U.S. Congress as well as California. Major food companies - including Frito-Lay, Heinz and Gerber - have announced plans to exclude transgenic ingredients from some of their products.

Biosafety Protocol. The first international treaty regulating trade in transgenic products, the United Nations Biosafety Protocol, was adopted in early 2000 by more than 130 nations including the United States. The Protocol seeks to "minimize the risks that biotechnology poses to the planet's diverse natural resources by regulating trade in living modified organisms." It requires that any international shipments of bioengineered bulk commodities, such as corn or soybeans, be accompanied by documentation stating that they "may contain" living modified organisms.

National Academy of Sciences (NAS) report. In April 2000, the NAS Committee on Genetically Modified Pest-Protected Plants issued its report on the science and regulation of transgenic crops. The report stressed there is no evidence that genetically engineered foods pose unique risks compared with those that are conventionally bred. The report also acknowledged that genetic engineering could improve plant health and agricultural productivity, and lessen the need for chemical pesticides. However, the committee identified ways that the research regimen for testing transgenic crops and current regulatory framework could be strengthened.

"There is an urgency to complete the regulatory framework for transgenic, pest-protected plant products because of the potential diversity of novel traits that could be introduced by transgenic methods and because of the rapid rate of adoption and public controversy regarding transgenic crops," the committee wrote.

The committee recommended that:

- Priority should be given to improving methods for identifying potential allergens.

- The impacts on nontarget organisms should be determined, compared with impacts of standard and alternative agricultural practices, through rigorous field evaluations.

- U.S. Environmental Protection Agency (EPA) should reconsider its categorical exemption of transgenic pest-protectants derived from sexually compatible plants; EPA should not categorically exempt viral coat proteins from regulation.

- U.S. Food and Drug Administration (FDA) should put a high priority on finalizing and releasing preliminary guidance on the assessment of potential food allergens.

- EPA, FDA and the U.S. Department of Agriculture, which regulate transgenics, should improve coordination and establish a process for timely information exchanges.

- The quantity, quality and public accessibility of information on the regulation of transgenic plants should be expanded.

Government oversight. On May 3, FDA announced plans to refine its regulatory approach regarding foods derived through the use of biotechnology. FDA plans to publish a proposed rule mandating that developers of bioengineered foods and animal feeds notify the agency 120 days before they intend to market new products, and requiring specific information to help determine whether they pose any potential safety, labeling or adulteration issues.

Public-interest group actions. On May 27, a coalition of 50 consumer, environmental, scientific, farm and health groups filed suit against FDA to obtain mandatory safety testing and labeling of genetically engineered foods. The suit alleges that current FDA policy violates the agency s mandate to protect public health and provide consumers with relevant information.
In January 2000, demonstrators marched in Montreal to protest genetlcally modified organisms (GMOs) during the United Nations Convention of Biological Diversity. The talks resulted in the Biosafety Protocol, an international treaty governing the movement of GMOs used in crops and foods. 